POS $\quad \begin{aligned} & \text { PROCEEDINGS } \\ & \text { OF SCIENCE }\end{aligned}$

\title{
A metric theory of gravity with torsion in extra-dimension
}

\author{
Kameshwar Wali* \\ Syracuse University \\ E-mail: waliephysics.syr.edu
}

A metric theory of gravity with torsion hidden in an extra-dimension is presented. With a set of constraints, non-vanishing components of torsion and connection coefficients are expressed in terms of the metric components resulting in modified Einstein equations, whose consequences on homogeneous, isotropic cosmology and static, spherically symmetric vacuum solutions are investigated.

36th International Conference on High Energy Physics,

July 4-11, 2012

Melbourne, Australia

\footnotetext{
* Speaker.
} 


\section{Introduction}

Einstein viewed the space-time as a pseudo-differential manifold in order to generalize the special-relativistic flat space-time to include gravity. The generalization not only implemented naturally the principle of equivalence, but it also had the revolutionary idea that the trajectory of any freely moving test body was simply a geodesic in the curved space-time manifold. Gravity was no longer a Newtonian instantaneous action-at-a-distance force.

Two basic ingredients of such a manifold are metric $g_{i j}$ and connection coefficient $\tilde{\Gamma}_{j k}^{i}$. Metric defines the distance between any two points and the connection leads to the definition of covariant derivative and the curvature in the manifold. The condition that the metric is covariantly constant (metric compatibility) leads to Eqns. 1.1 and 1.2 that determine the connection, $\tilde{\Gamma}_{j k}^{i}$, in terms of the metric and its derivatives (Christofell) and Contorsion $K_{j k}^{i}$ which contains what is called torsion, made up of the anti-symmetric combination of the connection coefficients.

$$
\begin{aligned}
\hat{\Gamma}_{j k}^{i} & =\frac{1}{2} \mathbf{g}^{i m}\left(\partial_{j} \mathbf{g}_{k m}+\partial_{k} \mathbf{g}_{j m}-\partial_{m} \mathbf{g}_{j k}\right), \\
K_{j k}^{i} & =\frac{1}{2}\left(T_{j k}^{i}+T_{j k}^{i}+T_{k j}^{i}\right),
\end{aligned}
$$

where

$$
\begin{aligned}
& T_{j k}^{i}=\tilde{\Gamma}_{j k}^{i}-\tilde{\Gamma}_{k j}^{i}, \\
& \text { and } \\
& \tilde{\Gamma}_{j k}^{i}=\hat{\Gamma}_{j k}^{i}+K_{j k}^{i} .
\end{aligned}
$$

In Einstein's GR, torsion is set to zero. Connection is then a unique function of just the metric. In view of the enormous success of GR, torsion is generally considered as superfluous. However, torsion becomes an integral part of any relativistic theory that includes intrinsic spinning matter - a more general theory, namely, Einstein-Cartan theory.

In this report, we will discuss briefly a novel approach to derive modified Einstein equations by including torsion within the framework of extra- dimensional theories of the Kaluza-Klein type. With a set of constraints, torsion is confined to the extra-dimension and dependent only on the metric. We consider a 5D manifold, foliated by a family of 4D surfaces. The axis of foliation, $x^{5}$ is special in the sense that there could exist non-vanishing torsion components along that dimension.

Consider a 5D manifold, foliated by a family of 4D surfaces (Fig.1). The axis of foliation, $x^{5}$ is special in the sense that there could exist non-vanishing torsion components along that dimension. The coordinates of the 5D manifold are denoted by the latin indices, $i, j, k, \ldots$ that take on values $0,1,2,3$ and 5 . And the coordinates along the $4 \mathrm{D}$ hypersurfaces by the Greek indices $\mu, \nu, \lambda, \ldots$ that take the values $0,1,2$, and 3 .

The basic formalism: 5D Metric $\left(x^{\mu}, x^{5}\right)$

$$
\begin{array}{r}
\mathbf{g}_{\mu v}=g_{\mu v}+\varepsilon A_{\mu} A_{v} \Phi^{2}, \mathbf{g}_{\mu 5}=\varepsilon A_{\mu} \Phi^{2}, \mathbf{g}_{55}=\varepsilon \Phi^{2} \\
\mathbf{g}^{\mu v}=g^{\mu v}, \mathbf{g}^{\mu 5}=-A_{\mu}, \mathbf{g}^{55}=A_{\lambda} A^{\lambda}+\varepsilon \Phi^{-2}
\end{array}
$$




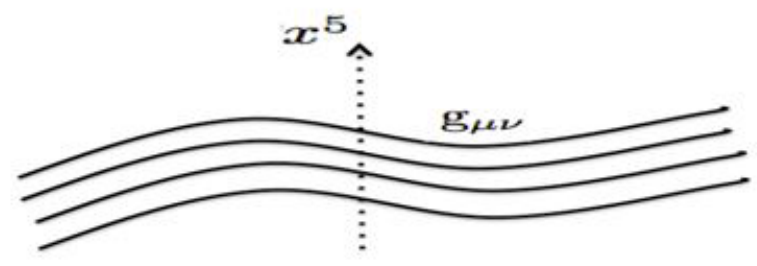

Figure 1: $x^{5}$

Note that the 5D metric is denoted by the bold face $\mathbf{g}$ and the 4D metric is denoted by the light faced $g$.

\section{Constraints and their implications}

The two constraints

$$
T_{\mu \nu}^{\lambda}=0, \quad \tilde{\Gamma}_{i 5}^{\lambda}=\tilde{\Gamma}_{5 i}^{\lambda}=0 .
$$

The first constraint implies the 4D Connection is symmetric as in GR. No torsion. The second arises by looking at the $4 \mathrm{D}$ components of geodesic equations in the $5 \mathrm{D}$ geometry and requiring that geodesic motion and its observable effects in 4D are not affected by any motion in the fifth dimension. The above two conditions are sufficient to determine uniquely the non-vanishing torsion components and the connection coefficients in terms of the metric:

$$
\begin{aligned}
T_{i j}^{\mu} & =T_{i j}^{5}=0, \\
T_{\mu v}^{5} & =2 \partial_{[\mu} A_{v]}+2 J_{[\mu} A_{v]}, \\
T_{\mu 5}^{5} & =J_{\mu}-\partial_{5} A_{\mu}-A_{\mu} J_{5} .
\end{aligned}
$$

And the connection coefficients are,

$$
\begin{aligned}
\tilde{\Gamma}_{55}^{\lambda} & =\tilde{\Gamma}_{v 5}^{\lambda}=\tilde{\Gamma}_{5 v}^{\lambda}=0, \\
\tilde{\Gamma}_{\mu v}^{5} & =\nabla_{\mu} A_{v}+J_{\mu} A_{v}, \\
\tilde{\Gamma}_{5 \mu}^{5} & =\partial_{5} A_{\mu}+J_{5} A_{\mu}, \\
\tilde{\Gamma}_{\mu 5}^{5} & =J_{\mu}, \quad \tilde{\Gamma}_{55}^{5}=J_{5}, \quad \tilde{\Gamma}_{\mu v}^{\lambda}=\Gamma_{\mu v}^{\lambda} .
\end{aligned}
$$

Here, $J_{i} \equiv \Phi^{-1} \partial_{i} \Phi$. In addition to determining the torsion and the connection coefficients in terms of the metric fields, the constraints also imply that the 4D metric on all the hypersurfaces are identical. As a consequence the 4D connection are also independent of $x^{5}$.

$$
\frac{\partial g_{\mu v}}{\partial x^{5}}=0 \Longrightarrow \frac{\partial \tilde{\Gamma}_{\mu \nu}^{\lambda}}{\partial x^{5}}=0 .
$$

This remarkable result is in contrast to the Kaluza-Klein theory, where it is a priori assumed that $g_{\mu \nu}, A_{\mu}$ and $\Phi$ are independent of $x^{5}$, which is known as the cylindrical condition. 


\section{Einstein Equations}

Substituting the non-vanishing connection coefficients, we find the Ricci tensor and Ricci scalar,

$$
\tilde{R}_{\mu v}=R_{\mu v}, \quad \tilde{R}_{\mu 5}=R_{5 \mu}=R_{55}=0
$$

They are exactly as in the torsion free $4 \mathrm{D}$ geometry. An important point to emphasize is that at the level of geometry, this framework is virtually indistinguishable from the torsion-free 4D spacetime of GR. Any observable geodesic motion or geodesic deviations between particles would match what we expect based on GR. However, this is true only to the extent the metric $\mathrm{g}$ is identical to the solution of the Einstein's equations in GR. However, this is indeed true only in the weak field limit for spherically symmetric vacuum solutions that are relevant for experimental observations within the solar system.

\section{Action Principle and Modified Einstein Equations}

We start with the standard Einstein-Hilbert action with the Ricci scalar as the Lagrangian density,

$$
S=\int d^{5} x \tilde{R} \sqrt{-g} .
$$

In varying the action, we note that Ricci scalar and the connection coefficients are functions of the metric components alone.

$$
\delta S=\int d^{5} x\left[\tilde{R} \delta \sqrt{-g}+\tilde{R}_{i k} \delta g^{i k} \sqrt{-g}\right]+\int d^{5} x\left[\delta \tilde{R}_{i k} g^{i k} \sqrt{-g}\right] .
$$

The first term in Eqn. 4.2 yields the usual Einstein tensor,

$$
\tilde{G}_{i k}=\tilde{R}_{i k}-\frac{1}{2} g_{i k} \tilde{R}
$$

leading to the standard Einstein equations. If torsion is absent, the second term becomes a boundary integral which vanishes when the variation is fixed at the boundary and does not contribute to the equations of motion. In the present case, torsion is present, but it is not an independent variable as in the general Einstein-Cartan theory, but expressed in terms of the metric. Varying the metric, we obtain the Modified Einstein eqns:

$$
\begin{aligned}
R_{\mu}^{v}-\frac{1}{2} R \delta_{\mu}^{v}+H_{\mu}^{v} & =\Sigma_{\mu}^{v}, \\
-A^{\alpha} R_{\mu \alpha}-A^{\alpha} H_{\mu \alpha} & =\Sigma_{5}^{\mu}, \\
-\frac{1}{2} R & =\Sigma_{5}^{5}
\end{aligned}
$$


with the additional terms involving the metric components of 5D space given by

$$
\begin{aligned}
H_{\mu v} & =\nabla_{(\mu} B_{v)}-(\nabla . B) g_{\mu v}+J_{(m u} B_{v)}-(J . B) g_{\mu v} \\
B_{\mu} & \equiv T_{\mu 5}^{5}=J_{\mu}-\partial_{5} A_{\mu}-A_{\mu} J_{5} .
\end{aligned}
$$

We note, since by construction, the fifth dimension is hidden with respect to the observable 4D motion, the 5D components of the stress energy tensor $\Sigma_{\mu}^{5}$ and $\Sigma_{5}^{5}$ are unobservable. Hence, in the present framework, only the first of the three Einstein equations 4.4 with the observable 4D stress tensor is relevant for solutions of physical interest.

\section{Homogenous and Isotropic Cosmology}

The 4D metric for a homogeneous and Isotropic cosmology of the universe has the form

$$
d s^{2}=-d t^{2}+a^{2}(t)\left(\frac{d r^{2}}{1-k r^{2}}+r^{2} d \Omega^{2}\right)
$$

The values of $\mathrm{k}=0,+1,-1$ correspond respectively to flat, closed, and hyperbolic spatial geometries.

In deriving the equations for $a(t)$ using modified Einstein equations, we shall assume the cylindrical condition that all the metric coefficients are independent of $x^{5}$ and further assume the stress tensor $\Sigma_{\mu}^{v}$ is conserved, although it does not have to satisfy the 4D conservation law when $H_{\mu}^{v}$ is non-vanishing. However, with minimum modifications to GR and the empirical conservation laws in mind, in what follows we shall assume the conservation law that leads to the conservation of $H_{\mu}^{v}$ and hence the relation

$$
\nabla_{v} H_{\mu}^{v}=3 J_{t}\left[\frac{\ddot{a}}{a}-\frac{\dot{a}}{a} J_{t}\right]=0
$$

This implies only two possibilities

$$
J_{t}=0 \text { or } J_{t}=\frac{\ddot{a}}{\dot{a}}
$$

From the definition of $J_{\mu}$, the first implies $\Phi$ is a constant, which would give rise to the usual Friedman-Robertson-Walker cosmology. The other alternative in Eqn. 5.2 combined with Eqns. 4.7 and 4.8 leads to

$$
\begin{aligned}
H_{t}^{t} & =3 \frac{\ddot{a}}{a}, \\
H_{r}^{r} & =2 \frac{\ddot{a}}{a}+\frac{\dddot{a}}{\dot{a}} .
\end{aligned}
$$

Taking the stress tensor to be that of a perfect fluid, the modified Einstein equations take the form 

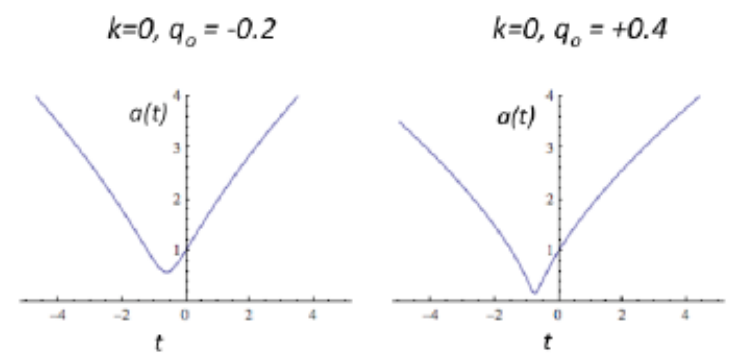

$$
k=0, q_{o}=+0.5
$$
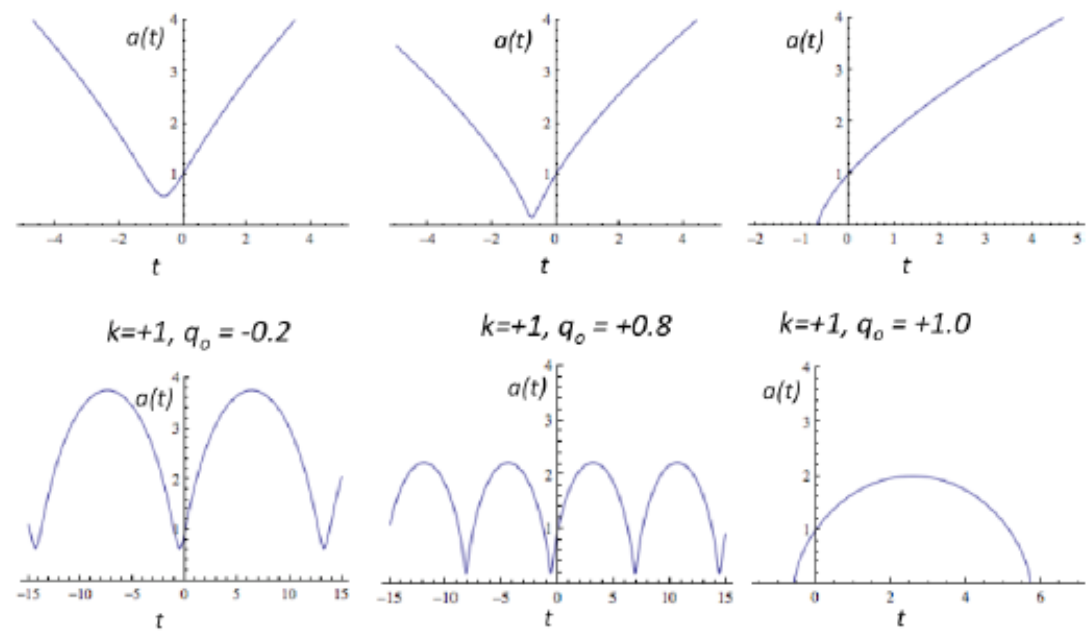

Figure 2: Solutions for $a(t)$ for different values of deceleration parameter for $k=0$ and $k=+1$.

$$
\begin{gathered}
3 \frac{\dot{a}^{2}}{a}+3 \frac{k}{a^{2}}+3 \frac{\ddot{a}}{a}=8 \pi \rho, \\
4 \frac{\ddot{a}}{a}+\frac{\dot{a}^{2}}{a}+\frac{\dddot{a}}{\dot{a}}+\frac{k}{a^{2}}=-8 \pi P .
\end{gathered}
$$

where $\rho$ and $P$ are the density and pressure of the 4D matter. The above two equations are sufficient to solve for $a(t)$. Without any loss of generality, we chose the needed two initial conditons; the current epoch to be set at $t=0$, the current size of the universe $a(0)=1$, and the unit time such that $\dot{a}(0)=1$. In this unit of time, the current value of the Hubble constant will be 1 . The effective equation for $a(t)$ then takes the form for matter dominated universe $(P=0)$

$$
\begin{aligned}
a^{2} \ddot{a}+a(\dot{a})^{2}+k a & =1+k-q_{0}, \\
q_{0} & =-\frac{a(0) \ddot{a}(0)}{\dot{a}(0)^{2}},
\end{aligned}
$$

where $q_{0}$ is the current value of the deceleration parameter, the only parameter that has to be specified.

Fig. 2 shows the behavior of $a(t)$ for various values $q_{0}$ for spatially flat and closed topologies. For spatially flat topology, shown in the top panels of the figure, we find that the universe does not originate from a singular big bang for all $q_{0}<+0.5$. For the spatially closed topology shown in the bottom panels, we find oscillatory solutions for all $q_{0}<+1$, which oscillate between $a_{\max }$ and $a_{\min }$. The acceleration reaches a ppositive value in a narrow interval around $a_{\min }$ and then becomes negative for the rest of the cycle until it gets back near $a_{\min }$. By taking the value of $q_{0}$ 
arbitrarily close to 1 , we can make $a_{\min }$ arbitrarily close to zero, as can be seen from the bottomleft and bottom-middle panels of the figure. Thus one could construct a universe that collapses and bounces back to expand when it reaches an arbitrarily small size, or equivalently arbitrarily high energy densities. It remains to be seen whether such solutions would fit the empirical redshift data.

\section{Static Spherically Symmetric Vacuum Solutions}

The most general 4D metric in this case has the form

$$
d s^{2}=-A(r) d t^{2}+B(r) d r^{2}+r^{2} d \Omega^{2},
$$

which when coupled with the modified Einstein equations leads to the following coupled equations:

$$
\begin{aligned}
J^{\prime} & =-\frac{J(1+B)}{r}, \\
A^{\prime} & =-\frac{2 A(1-B+2 r J)}{r(2+J r)}, \\
B^{\prime} & =\frac{2 B\left(r^{2} J^{2}+(1+r J)(1-B)\right)}{r(2+J r)} .
\end{aligned}
$$

In deriving the above equations, we have used the cylindrical condition that gives

$$
H_{\mu}^{v}=\nabla_{\mu} J^{v}-(\nabla . J) \delta_{\mu}^{v}+J_{\mu}^{v}-(J . J) \delta_{\mu}^{v} .
$$

and then the additional terms to Einstein equations follow.

$$
\begin{aligned}
H_{t}^{t} & =\frac{r J B^{\prime}-2 B\left(r J^{\prime}+2 J+r J^{2}\right)}{2 r B^{2}}, \\
H_{r}^{r} & =-\frac{J\left(r A^{\prime}+4 A\right)}{2 r A B}, \\
H_{\theta}^{\theta} & =\frac{r J A B^{\prime}-2 r A B J^{\prime}-r J B A^{\prime}-2 A B J(1+r J)}{2 r A B^{2}}, \\
H_{\phi}^{\phi} & =H_{\theta}^{\theta} .
\end{aligned}
$$

Some general remarks from Eqn. 6.3:

1. If $\mathrm{J}(\mathrm{r})$ is a constant, it has to be identically zero

2. If $\mathrm{J}(\mathrm{r})$ vanishes at some point, it has to vanish everywhere. The simplest solution

$$
J(r)=0, A(r)=\left(1-\frac{2 M}{r}\right), B(r)=\left(1-\frac{2 M}{r}\right)^{-1}
$$

is the well-known Schwarzschild solution, as expected. 

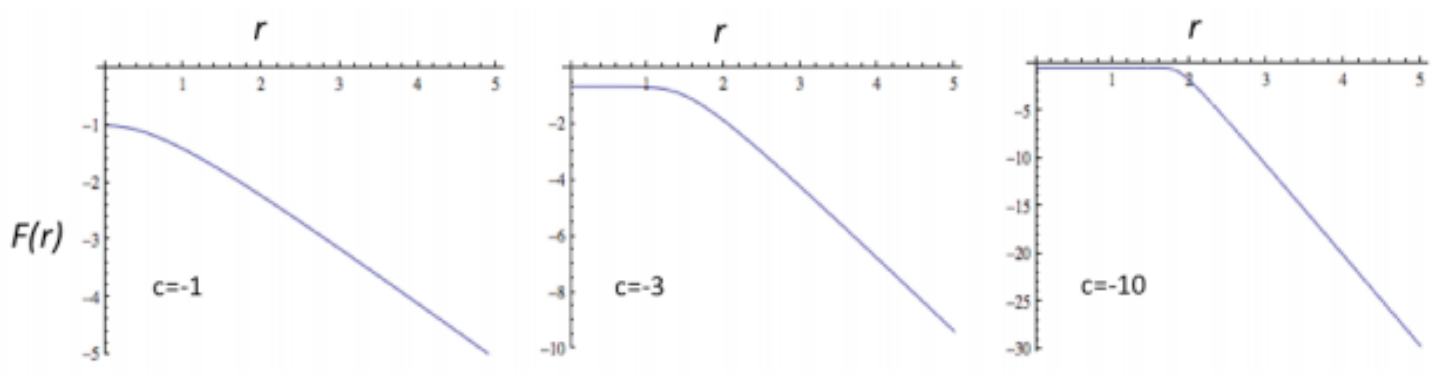

Figure 3: Numerical solutions for $F(r)$ with $\lambda=-1$

When $J(r)$ is not equal to zero and not a constant, with $r F=1 / J(r)$, we obtain, after some algebra, a second order non-linear equation,

$$
r\left[\left(2 F^{2}+F\right) F^{\prime \prime}+F^{2}\right]=F^{\prime}(F+2),
$$

whose solution in an implicit form is given by

$$
\lambda^{2} r^{2}=\gamma^{2}|F / \gamma+\beta+1|^{1+\beta}|F / \gamma+\beta-1|^{1-\beta},
$$

where,

$$
\gamma=\sqrt{1+c+c^{2}}, \beta=\frac{1+c}{\sqrt{1+c+c^{2}}} .
$$

and $\mathrm{c}$ is an arbitrary constant.

The metric functions $A(r)$ and $B(r)$ are given in terms of $F(r)$ :

$$
\begin{aligned}
& A(r)=\exp \left(-\int_{r}^{\infty} d r \frac{2 c}{r F(r)}\right), \\
& B(r)=1+\frac{2(1+c)}{F(r)}+\frac{c}{F^{2}(r)} .
\end{aligned}
$$

A careful study of the dependence of the numerical solutions on the parameters leads one to conclude:

1. For $c>0, F$ is negative definite and $\lambda$ is negative .

2. For $c<0, F$ is positive definite with $\lambda$ positive or negative.

The qualitative behavior of the functions $A(r)$ and $B(r)$ are shown in Fig. 4 for various allowed ranges of $c$ and $\lambda$. When $M=\left[c \lambda^{-1}\right]$ is positive, $A(r)$ is a monotonically increasing function leading to attractive gravity, and it corresponds to a Schwarzschild solution at large $r$ when $c>>1$.

An important point to note from the figure is that these solutions do not have an event horizon because both $A(r)$ and $B(r)$ are finite and positive for all $r>0$. Clearly, these solutions are smooth for all $r>0$. However, the point $r=0$ is a physical singularity. Explicit calculations show that the Ricci scalar $\mathrm{R}$ vanishes everywhere, but the quantity $R_{\mu \nu} R^{\mu v}$ is nonvanishing and it diverges at $r=0$. 

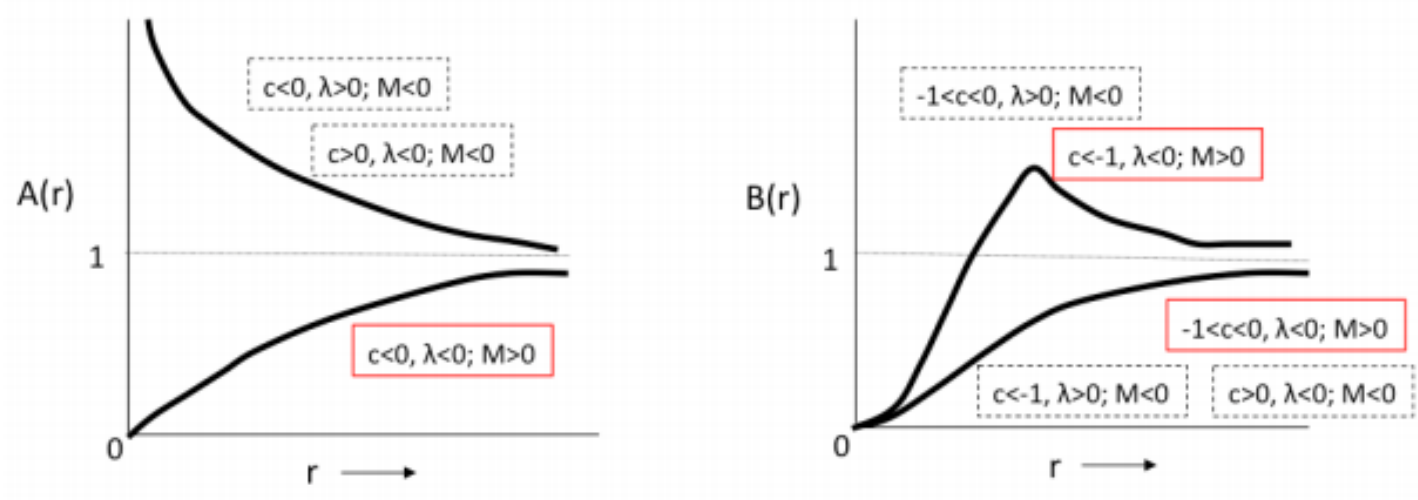

Figure 4: Schematic behavior of $A(r)$ and $B(r)$

\section{Summary and Conclusions}

Metric and torsion are two independent constituents of metric compatible Riemannian geometry. Because of the immense successes of GR torsion has not played a significant role in theories of relativity. However, when gravity is to be included with other elementary particle interactions with intrinsic spin, a more general theory of including torsion becomes imperative. In the present work, torsion is incorporated in a novel way in higher dimensional Kaluza-Klein theories. Here torsion is not an independent degree of freedom coupled to spin, rather it is determined in terms of metric through a set of constraints, which serve to confine torsion to the extra dimension, leaving the 4D space-time torsion free. The resulting modified Einstein equations, however, have significant physical consequences. In the case of Isotropic-homogeneous cosmology, one has different possibilities other than the big bang standard FRW cosmology. The modified second order differential equation replacing the conventional FRW equation allows one to choose the initial condition for the second time derivative of $a(t)$, which can be tuned to the observed acceleration of the universe. In FRW cosmology no such possibility exists, resulting in a decelerating expansion in the absence of a cosmological constant.

In the case of spherically symmetric vacuum solutions, the existence of positive mass solutions with naked singularity have immediate consequences on gravitational collapse, opening up the possibility of an arbitrarily large star collapsing to an arbitrarily small non-singular state. Since trapped surfaces would not necessarily form in such collapses, finite matter pressure may be sufficient to withstand the singularity. This and other aspects of the work need further investigation.

This work was done in collaboration with Karthik H. Shankar and Anand Balaraman.

\section{Acknowledgements}

The work was supported in part by U.S. Department of Energy (DOE)-DE-FG02-85ER40237 (K.C.Wali) 


\section{References}

[1] Shankar, Karthik H. and Balaraman, Anand and Wali, Kameshwar C., A Metric theory of gravity with torsion in extra-dimension, Phys.Rev.D 86 (024007) [1203.5552]. 